\title{
A Case of Deep Vein Thrombosis Caused by Hematoma Following Total Knee Arthroplasty
}

\author{
Takenori Tomite ${ }^{1,4, *}$, Hidetomo Saito ${ }^{2,4}$, Hiroaki Kijima ${ }^{2,4}$, Kimio Saito ${ }^{2,4}$, Noriyuki Ishikawa ${ }^{1}$, \\ Yuji Hatakeyama $^{1}$, Hiroshi Tazawa ${ }^{1}$, Satoshi Yumoto ${ }^{1}$, Ryo Syoji ${ }^{1}$, Toshiaki Aizawa ${ }^{3}$, \\ Naohisa Miyakoshi ${ }^{2}$, Yoichi Shimada ${ }^{2,4}$ \\ ${ }^{1}$ Department of Orthopedic Surgery, Akita Red Cross Hospital, Akita, Japan \\ ${ }^{2}$ Department of Orthopedic Surgery, Akita University Graduate School of Medicine, Akita, Japan \\ ${ }^{3}$ Department of Orthopedic Surgery, Kitaakita Municipal Hospital, Akita, Japan \\ ${ }^{4}$ Akita Sports, Arthroscopy, and Knee Group (ASAKG), Akita, Japan
}

Email address:

takenoritomite@yahoo.co.jp (T. Tomite)

${ }^{*}$ Corresponding author

\section{To cite this article:}

Takenori Tomite, Hidetomo Saito, Hiroaki Kijima, Kimio Saito, Noriyuki Ishikawa, Yuji Hatakeyama, Hiroshi Tazawa, Satoshi Yumoto, Ryo Syoji, Toshiaki Aizawa, Naohisa Miyakoshi, Yoichi Shimada. A Case of Deep Vein Thrombosis Caused by Hematoma Following Total Knee Arthroplasty. Clinical Medicine Research. Vol. 7, No. 5, 2018, pp. 131-134. doi: 10.11648/j.cmr.20180705.15

Received: September 19, 2018; Accepted: October 26, 2018; Published: November 28, 2018

\begin{abstract}
Deep vein thrombosis is one of the main complications of total knee arthroplasty. Hematoma is another complication often seen following total knee arthroplasty, but a search of the literature found no reports of deep vein thrombosis caused by hematoma. A case of a 68-year-old woman with knee osteoarthritis who underwent total knee arthroplasty, with subsequent onset of thrombosis caused by hematoma, is reported. The surgery was performed through the medial parapatellar approach with measured resection and the implant was put in place with cement fixation. On postoperative day 7, there was still leakage of exudate from the wound, and swelling of the lower leg appeared. Therefore, infection or thrombosis was suspected, and contrast-enhanced computed tomography was performed. Accumulation of fluid was found from the thigh to the knee, and when paracentesis was carried out, blood was drawn, resulting in a diagnosis of hematoma. In addition, the blood vessels were under pressure from the hematoma, and thrombosis was diagnosed based on the clinical, blood analysis, and ultrasound findings. There was concern about treatment due to coexistence of hematoma and thrombi, but through application of a Robert Jones bandage and administration of edoxaban, the patient made good progress with no major complications. A case of deep vein thrombosis caused by hematoma following total knee arthroplasty was reported. The patient recovered without major complications through administration of edoxaban and application of a Robert Jones bandage.
\end{abstract}

Keywords: Total Knee Arthroplasty, Deep Venous Thrombosis, Anticoagulant

\section{Introduction}

Total knee arthroplasty (TKA) is one of the most effective methods of treatment for end-stage knee osteoarthritis. However, various complications are associated with TKA, and one main complication is deep vein thrombosis (DVT). It has been reported that, if appropriate management is not carried out following TKA, the incidence of DVT is $40 \%$ to $80 \%$, and the incidence of pulmonary embolism is approximately $2 \%$ [1]. There is, therefore, a consensus that measures to prevent thrombus formation are essential. In addition, hematoma formation following TKA is also a known complication that occurs with high frequency [2], but there are no reports of cases of DVT occurring as a result of hematoma or of methods to manage hematoma and thrombus that occur simultaneously.

In addition, while various guidelines for the treatment of thrombi have been published [8-10], there are no guidelines for coping with situations in which hemorrhage is a concern. Cases such as the present case in which DVT is caused by a large hematoma are probably rare, but situations in which hemorrhage is a concern while at the same time there are also worries about thrombi, are sometimes encountered in 
everyday clinical practice. Direct oral anticoagulants (DOACs) have been included in health insurance coverage in recent years in Japan, and safe, useful treatments for thrombi have started to be used, but there appear to be few reports of such treatments in situations in which hemorrhage is a concern. A case of DVT caused by hematoma following TKA is reported, along with a discussion of the literature.

\section{Case Presentation}

The patient was a 68-year-old woman with pain in both knees that commenced in 2008. Despite conservative treatment, the pain in her right knee increased, and she underwent right TKA in May 2015. Her past medical history included hypertension and insomnia, and she was taking valsartan, amlodipine besilate, brotizolam, and celecoxib. There was no history of either blood disease or trauma to the knees. Prior to surgery, the range of motion of the right knee was $-15^{\circ}$ to $90^{\circ}$, with no hydrarthrosis. The preoperative $\mathrm{X}$-ray showed degenerative changes (Figure $1 \mathrm{~A}, \mathrm{~B}$ ).

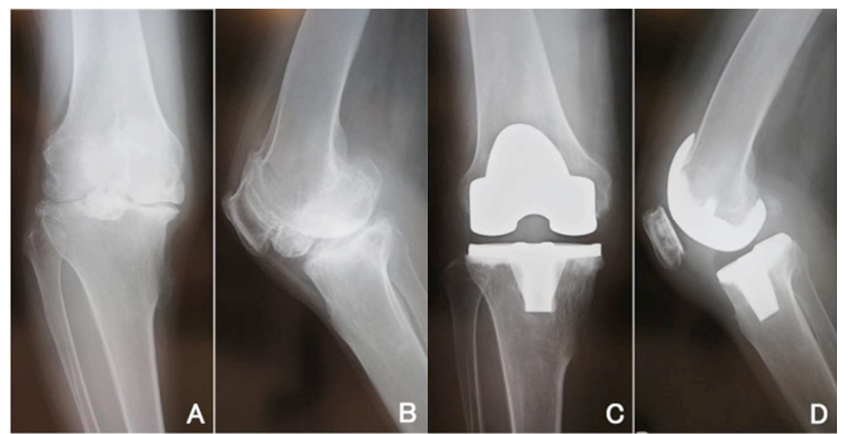

Figure 1. Frontal (Figure. 1A) and lateral (Figure. 1B) preoperative X-rays, and frontal (Figure. 1C) and lateral (Figure. 1D) postoperative X-rays.

The TKA operative procedure was performed through a medial parapatellar incision, the implant was a posterior stabilized Persona (Zimmer Biomet Holdings, Inc. Warsaw, IN, USA), which was put in place with cement fixation, and the patella was resurfaced. Following implant placement, the tourniquet was opened, and hemostasis was performed. Tourniquet time was 55 minutes. When it was confirmed that there was no bleeding, a drain was placed and the wound was closed. The postoperative X-ray showed that the implant was placed with no alignment problems (Figure. 1C, 1D).

Continuous passive motion commenced from postoperative day 1 , and on postoperative day 2 , the drain was removed and transfers from the bed to a wheelchair commenced. On postoperative day 3 , there was leakage of serosanguinous exudate from the wound. This was considered to be leakage of hematoma within the knee joint, and, therefore, her progress was monitored with no anticoagulant therapy carried out. On postoperative day 7, there was still leakage of exudate from the wound, and the swelling from the thigh to the lower leg had increased. Therefore, infection or thrombosis was suspected, and contrast-enhanced computed tomography (CT) was performed. Images of accumulated fluid mixed with air were found from the knee to the thigh, and the fluid accumulation resulted in displacement of vessels deep in the thigh (Figure. 2A, 2B). When paracentesis was performed at the lateral thigh under ultrasound guidance, blood was drawn. Bacteria were not detected in a subsequent test of synovial fluid, and on an ultrasound scan of the lower leg carried out on the same day, thrombi were found at a medial branch and a middle branch of the soleal vein (Figure 3A, 3B). The D-dimer values were $0.68 \mu \mathrm{g} / \mathrm{ml}$ preoperatively and 27.98 $\mu \mathrm{g} / \mathrm{ml}$ on postoperative day 6 .

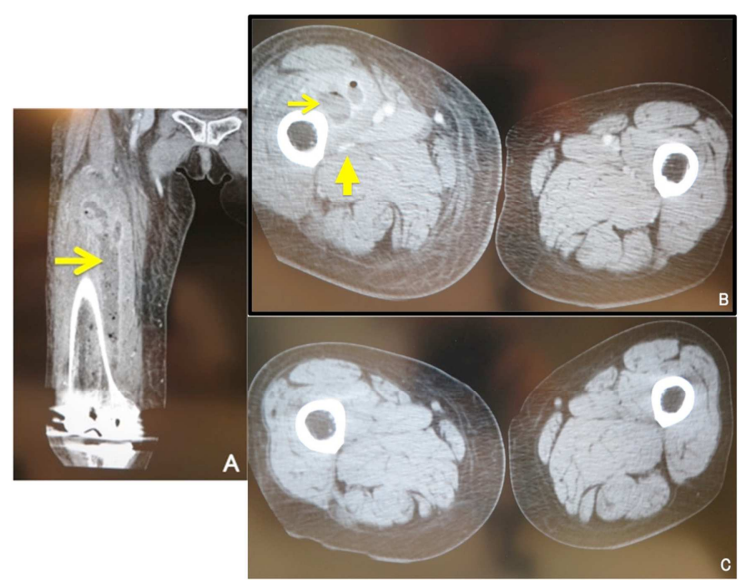

Figure 2. Contrast-enhanced CT scans on postoperative day 6 (Figure 2A, $2 B$ ). Accumulated fluid mixed with air is found from the knee to the thigh (fine arrows). There is also marked swelling of soft tissues in comparison to the left knee, and the vessel is displaced and flattened due to the presence of hematoma (thick arrow). CT scan on postoperative day 20 (Figure 2C). The hematoma has been absorbed, and the soft tissue swelling has abated.
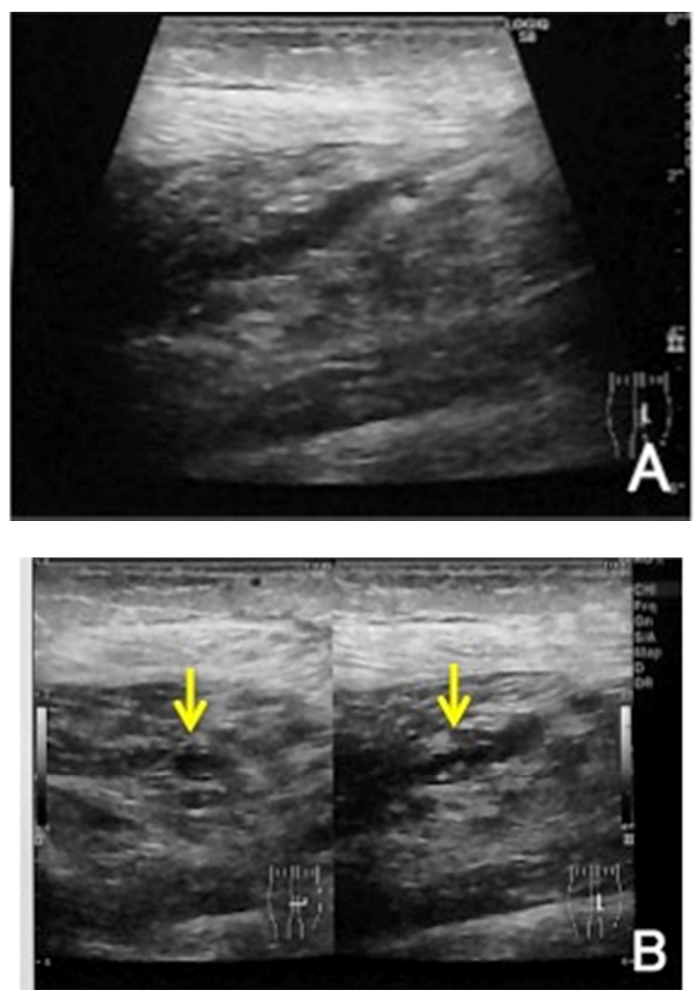

Figure 3. Ultrasound scan of the lower leg on postoperative day 6. Beaded dilation of the soleal vein can be seen (Figure $3 A$ ). Inside the vein, images of isoechoic to partially high-echoic thrombi are seen (Figure 3B). The vein inside which these thrombi were found is not crushed by pressure. 
Since thrombi were found, anticoagulant therapy seemed preferable, but there was reluctance to administer strong anticoagulant therapy because of the presence of the hematoma. A Robert Jones bandage (RJB) was applied to administer pressure from the thigh to the lower leg, and edoxaban administration commenced at a dose of $15 \mathrm{mg}$, which is half the normal dose (the recommended dose for a patient weighing less than $60 \mathrm{~kg}$ is $30 \mathrm{mg}$ [8-9]). On postoperative day 20, the swelling from the thigh to the lower leg had abated. An ultrasound scan showed that the thrombi were smaller, and CT showed that the hematoma was absorbed and the swelling abated (Figure 2C). In addition, the D-dimer value on postoperative day 20 was $4.36 \mu \mathrm{g} / \mathrm{ml}$.

As of one year postoperatively, the knee range of motion was $0^{\circ}$ to $115^{\circ}$, and the patient was able to walk unaided and had not experienced recurrence of hematoma or thrombus.

\section{Discussion}

Virchow's triad has long been known to describe factors responsible for deep vein thrombus formation, and with the addition to this theory of new knowledge, changes in blood flow, the blood itself, and the endothelium are currently also considered responsible [3-4]. In the present case, while there was general surgical invasion that would impair blood flow and the endothelium, no abnormalities of the blood itself that might cause thrombus or hematoma formation were found in platelet count, bleeding time, activated partial thromboplastin time, or prothrombin time.

In addition, the technique of managing postoperative drainage [5], use of a tourniquet [6], and positioning of the lower leg during surgery [7] have been reported as factors in hematoma formation following TKA, but the definite cause remains unclear. One limitation of the present case is that diseases that are predisposing factors for thrombus formation, such as antiphospholipid antibody syndrome and vasculitis syndrome, or diseases in which a bleeding tendency is seen despite the absence of blood test abnormalities such as platelet dysfunction were not ruled out.

Various guidelines have been published for the treatment of thrombus [8-10]. However, it has been pointed out that, since there are large differences between Western and Japanese people in the frequency of occurrence, and there are also differences between Japan and overseas countries in regard to drugs that are covered by health insurance, guidelines from other countries cannot be used unchanged in Japan. Since 2014, DOACs have been covered by health insurance for treatment of venous thromboembolism (VTE). In the HOKUSAI-VTE trial, a phase III clinical trial of edoxaban for VTE, edoxaban was reported to have the same level of effectiveness in preventing VTE recurrence as conventional therapy and to cause a significant reduction in serious bleeding events [11]. In a sub-analysis, edoxaban was also shown to have the same efficacy among East Asian populations, including the Japanese [12]. In the present case, the normal dose of $15 \mathrm{mg}$ edoxaban, which is half dose, were used because there was concomitant hematoma, and no aggravation of the hematoma was found.
The RJB has a long history, but the first written report of its use was by Sir John Charnley in 1950 [13]. The RJB is reported to reduce bleeding, to abate tissue swelling, and to decrease pain [13-14], and it is widely used as a postoperative or posttraumatic bandage. However, there is also a report that a modified RJB used following TKA showed no difference in the postoperative amount of bleeding, pain, or swelling when compared to a conventional wound dressing [15]. In the present study, even though edoxaban was administered, bleeding was controlled by application of an RJB.

\section{Conclusion}

In conclusion, a case of DVT caused by hematoma following TKA was reported. The patient recovered without major complications through administration of edoxaban and application of an RJB. In cases of thrombosis where hemorrhage is a concern following surgery to the leg, management of thrombi by control of bleeding with an RJP while edoxaban is administered may be useful.

\section{Funding}

The author(s) received no financial support for the research, authorship, and/or publication of this article.

\section{Declaration of Conflicting Interests}

The author(s) declare no potential conflicts of interest with respect to the research, authorship, and/or publication of this article.

\section{References}

[1] Mao Lin He, Zeng Ming Xiao, Ming Lei et al. Continuous passive motion for preventing venous thromboembolism after total knee arthroplasty. Cochrane Database Syst Rev. 2014;7:CD008207.

[2] Ning Liu, Simin Luo, Cheanglek Hang et al. Changes in coagulation functions and hemorheological parameters may predict hematoma formation after total knee arthroplasty. J Orthop Surg Res. 2016;11:35.

[3] Mackman N. New insights into the mechanisms of venous thrombosis. J Clin Invest. 2012;122:2331-2336.

[4] Schulz C, Engelmann B, Massberg S. Crossroads of coagulation and innate immunity: the case of deep vein thrombosis. J Thromb Haemost. 2013;11(Suppl 1):233-241.

[5] Esler CN, Blackeway C, Fiddian NJ. The use of a closed-suction drain in total knee arthroplasty. A prospective, randomized study. J Bone Joint Surg Br. 2003;85(2):215-7.

[6] Bin Li, Yu Wen, Haishan Wu et al. The effect of tourniquet use on hidden blood loss in total knee arthroplasty. Int Orthop. 2009;33(5):1263-8.

[7] Jun Liu, Yao-min Li, Jian-gang Cao et al. Effects of knee position on blood loss following total knee arthroplasty: a randomized, controlled study. J Orthop Surg Res. 2015;10:69. 
[8] Konstantinides SV, Torbicki A, Agnelli G et al. 2014 ESC guidelines on the diagnosis and management of acute pulmonary embolism. Eur Heart J. 2014;35:3033-3069.

[9] Kearon C, Akl EA, Comerota AJ et al. Antithrombotic therapy for VTE Disease: CHEST Guideline and Expert Panel Report. Chest. 2016;149(2):315-52.

[10] Kearon C, Akl EA, Ornelas J et al. Antithrombotic therapy for VTE disease: Antithrombotic Therapy and Prevention of Thrombosis, 9th de: American College of Chest Physicians Evidence-Based Clinical Practice Guidelines. Chest. 2012;141(2 Suppl):e419S-494S.

[11] Hokusai-VTE Investigations, et al. Edoxaban versus warfarin for the treatment of symptomatic venous thromboembolism. N Engl J Med. 2013;369:1406-1415.
[12] Nakamura M, Wang YQ, Wang C et al. Efficacy and safety of edoxaban for treatment of venous thromboembolism: a subanalysis of East Asian patients in the Hokusai-VTE trial. J Thromb Haemost. 2015;13:1606-1614.

[13] Brodell JD, Axon DL, Evarts CM. The Robert Jones bandage. J Bone Joint Surg Br. 1986;68:776-779.

[14] Charalambides C, Beer M, Melhuish J et al. Bandaging technique after knee replacement. Acta Orthop. 2005;76:89-94.

[15] Piya Pinsornsak, Sukanis Chumchuen. Can a modified Robert Jones bandage after knee arthroplasty reduce blood loss? A prospective randomized controlled trial. Clin Orthop Relat Res. 2013;471(5):1677-81. 